\title{
Partial Laryngopharyngectomy
}

National Cancer Institute

\section{Source}

National Cancer Institute. Partial Laryngopharyngectomy. NCI Thesaurus. Code C150662.

Laryngopharyngectomy in which one side of the larynx is spared from surgery. 